\title{
Autologous transplantation of adipose- derived stem cells improves functional recovery of skeletal muscle without direct participation in new myofiber formation
}

\author{
Agata Gorecka', Souzan Salemi ${ }^{2}$, Deana Haralampieva ${ }^{2}$, Federica Moalli4, ${ }^{4,5}$, Deborah Stroka ${ }^{1}$, Daniel Candinas ${ }^{1,3}$,
} Daniel Eberli ${ }^{2}$ and Lukas Brügger ${ }^{1,3^{*}}$ (D)

\begin{abstract}
Background: Skeletal muscle has a remarkable regenerative capacity. However, extensive damage that exceeds the self-regenerative ability of the muscle can lead to irreversible fibrosis, scarring, and significant loss of function. Adiposederived stem cells (ADSC) are a highly abundant source of progenitor cells that have been previously reported to support the regeneration of various muscle tissues, including striated muscles. The aim of this study was to evaluate the effect of ADSC transplantation on functional skeletal muscle regeneration in an acute injury model.

Methods: Mouse ADSC were isolated from subcutaneous fat tissue and transplanted with a collagen hydrogel into the crushed tibialis anterior muscle of mice. Recovering muscles were analyzed for gene and protein expression by realtime quantitative polymerase chain reaction and immunohistochemistry. The muscle contractility was assessed by myography in an organ bath system.

Results: Intramuscular transplantation of ADSC into crushed tibialis anterior muscle leads to an improved muscle regeneration with ADSC residing in the damaged area. We did not observe ADSC differentiation into new muscle fibers or endothelial cells. However, the ADSC-injected muscles had improved contractility in comparison with the collagen-injected controls 28 days post-transplantation. Additionally, an increase in fiber cross-sectional size and in the number of mature fibers with centralized nuclei was observed.
\end{abstract}

Conclusions: ADSC transplantation into acute damaged skeletal muscle significantly improves functional muscle tissue regeneration without direct participation in muscle fiber formation. Cellular therapy with ADSC represents a novel approach to promote skeletal muscle regeneration.

Keywords: Adipose-derived stem cells, Crush injury, Skeletal muscle regeneration, Stem cell therapy, Tissue engineering

\section{Background}

Skeletal muscle is the most common and widely distributed tissue in the human body $[1,2]$ composing approximately $40 \%$ of the total body weight. Structurally, it is built of parallel multinucleated fibers that contract upon stimulation, generating force [3]. The ability of skeletal

\footnotetext{
* Correspondence: lukas.bruegger@insel.ch

'Department of Clinical Research, Laboratory for Visceral Surgery and Medicine, University of Bern, Murtenstrasse 35, 3008 Bern, Switzerland ${ }^{3}$ University Clinic for Visceral Surgery and Medicine, Bauchzentrum Bern, Inselspital, CH-3010 Bern, Switzerland

Full list of author information is available at the end of the article
}

muscle to regenerate is highly dependent on endogenous paired box protein domain 7 (Pax7)-positive stem cells called satellite cells $(\mathrm{SC})[4,5]$ which reside under the basal lamina of each muscle fiber. Immediately upon stress or injury, SC are activated and re-enter the cell cycle. A small fraction of SC return to quiescent state; however, most of them migrate to the injury site as muscle precursor cells (MPC). MPC committed to myogenesis downregulate Pax7 expression and at the same time upregulate the transcription factor myogenic differentiation antigen $(\mathrm{MyoD})$. They further differentiate into

(c) The Author(s). 2018 Open Access This article is distributed under the terms of the Creative Commons Attribution 4.0 International License (http://creativecommons.org/licenses/by/4.0/), which permits unrestricted use, distribution, and 
myoblasts and myofibers, restoring missing muscle mass [6-9]. The mature myofibers express the characteristic structural proteins like myosin heavy chain ( $\mathrm{MyHC}$ ). This regenerative mechanism is very efficient in response to minor injuries; however, upon large damage the deposition of fibrotic tissue can exceed the intrinsic regenerative capacity, leading to impaired muscle flexibility and strength $[10,11]$. Although there are surgical treatment options available, in cases with major damage the outcomes are unsatisfactory [12-14].

Several cell-based therapies have been investigated as a possible treatment for severely damaged skeletal muscles [15-18]. Adipose-derived stem cells (ADSC) have received much attention within the last years, as they are an easily accessible source of stem cells for regenerative therapies. ADSC are mesenchymal stem cells (MSC) that can be isolated in large quantities from the perivascular area of subcutaneous fat tissue by liposuction [19-22]. They are a heterogeneous population of cells at different stages of maturation and differentiation and have broad plasticity and differentiation potential similar to MSC isolated from the bone marrow (BM-MSC) [23-27]. Moreover, the adipose tissue has a significantly higher stem cell density than bone marrow (5\% versus 0.01\%) [28]. Myogenic differentiation in vitro as well as in vivo of the ADSC has been reported so far in several studies [29-31]. Additionally, ADSC have been shown to support tissue regeneration in a paracrine manner by secreting soluble factors promoting cell survival and tissue regeneration $[32,33]$. The aim of this study was to evaluate the therapeutic potential of ADSC on the functional recovery of skeletal muscle.

\section{Methods}

\section{Cell isolation and culture}

All the animal experiments were performed according to Federation for Laboratory Animal Science Associations guidelines and approved by the Animal Care Committee of the Canton Bern, Switzerland (study no. BE 88/12).

The ADSC were isolated and cultured as described previously [34]. Briefly, the subcutaneous white fat pads were removed form 7- to 8-week-old female C57BL/6 mice (Envigo, Netherlands). Harvested fat was minced into small pieces and digested in $0.1 \%$ collagenase type I (Sigma-Aldrich, Buchs, Switzerland) solution at $37{ }^{\circ} \mathrm{C}$ for $1 \mathrm{~h}$. The samples were centrifuged, the supernatant containing adipocytes was discarded, and the stromal vascular fraction pellet was filtered $(100 \mu \mathrm{m})$ and cultured in a growth medium (Dulbecco's modified Eagle's medium (DMEM)/F12, 10\% fetal bovine serum (FBS), 1\% penicillin-streptomycin (PS); all from Gibco by Life Technologies, Carlsbad, USA). After $24 \mathrm{~h}$, the nonadherent cells were removed. Cells were passaged at $80-90 \%$ confluency. ADSC from passage 3 were used in all experiments.

The mouse SC were isolated as described previously [35]. Briefly, the EDL muscle was removed from the recently euthanized C57BL/6 (females, 5 weeks old) and digested in $0.2 \%$ collagenase I (Sigma-Aldrich) for 90 min. After digestion, the single myofibers were transferred to matrigel matrix (Corning)-coated plates (1:10 dilution in DMEM) and cultured in high-serum proliferation-inducing medium containing DMEM, $20 \%$ FBS, 10\% horse serum (HS), 1\% PS (Gibco by Life Technologies), $0.5 \%$ chicken embryonic extract (CEE; US Biologicals, Salem, MA), and $10 \mathrm{ng} / \mathrm{ml}$ basic fibroblast growth factor (bFGF; PeproTech, Rocky Hill, $\mathrm{NJ})$. SC upon activation migrate from the myofibers on the culture dish. Cells were passaged first time after 7 days and for every other passage at $60 \%$ confluency. To induce differentiation, SC were placed in a low-serum medium (DMEM, 2\% HS, 4\% FBS, 0.5\% CEE, 1\% PS).

For coculture experiments the SC were plated on matrigel-coated dishes with a cell density of $5 \times 10^{5}$ cells/ $\mathrm{cm}^{2}$ in proliferation medium. After reaching $60 \%$ confluency, the cells were serum starved for $24 \mathrm{~h}$ and then placed in coculture with/without ADSC seeded on the inserts $(0.4 \mu \mathrm{m}$; Greiner Bio-One, St. Gallen, Switzerland) in proliferation or differentiation medium. For the ADSC-conditioned (CM-ADSC) medium, SC were cultured in the same medium composition that was changed every $24 \mathrm{~h}$. After $72 \mathrm{~h}$ the cells were processed for real-time quantitative polymerase chain reaction (RT-qPCR) and Western blot (WB) analysis, and the SC in differentiation medium were additionally stained for fiber formation with Giemsa solution (Fluka Chemie, Buchs, Switzerland).

\section{Flow cytometry analysis}

Isolated cells were incubated with primary antibodies for 40 min at $4{ }^{\circ} \mathrm{C}$ in phosphate-buffered saline (PBS) supplemented with $2 \%$ FBS and $2 \mathrm{mM}$ ethylenediaminetetraacetic acid (EDTA). We used the following directly conjugated antibodies: CD29 (R\&D Systems), CD44 (BioLegend), CD90.2 (BioLegend), CD31 (BioLegend), CD34 (BioLegend), stem cell antigen 1 (Sca-1; e-Bioscience), PDGFR $\alpha$ (BioLegend), and PDGFR $\beta$ (BioLegend). All stainings were controlled with appropriate isotope control antibodies. Analysis was performed on a SORP LSRII (Becton Dickinson) equipped with five lasers and data were collected with FACS DIVA software. Analysis was performed using Flow) ${ }^{\text {Tw }}$ 10.0.8 (Treestar, Ashland, OR). All measurements were performed with three biological replicates.

\section{Immunocytochemistry}

Cells were fixed with $4 \%$ paraformaldehyde, permeabilized with $0.1 \%$ Triton-X and blocked with $5 \%$ bovine serum albumin (BSA) for $1 \mathrm{~h}$. Samples were incubated 
overnight at $4{ }^{\circ} \mathrm{C}$ with the primary antibodies $\mathrm{CD} 44$ (BD Pharmingen), CD29 (R\&D Systems), CD90.2 (BD Pharmingen), CD31 (Abcam, Cambridge, UK), MyHC (in house production), Pax7 (DSHB, Iowa, USA), and MyoD (Santa Cruz Biotechnology, Heidelberg, Germany). Primary antibodies were detected using goat anti-rabbit or goat anti-mouse IgG antibodies conjugated to fluorescein isothiocyanate (FITC) or Streptavidin Alexa 594. Cells were counterstained for F-actin with phalloidin-FITC (Sigma-Aldrich) or phalloidin-Atto 550 and for nuclear staining with DAPI (Sigma-Aldrich).

Cells were visualized using a fluorescence microscope (Nikon Eclipse 800) or confocal laser scanning microscope (Zeiss LSM 710; Carl Zeiss Microscopy, Oberkochen, Germany).

For fiber formation assay the cultured myofibers were fixed with methanol for $7 \mathrm{~min}$ and stained with Giemsa solution (1:20) for $1 \mathrm{~h}$ at room temperature. The number of myofibers per high-power field was calculated along with the number of nuclei per myofiber.

\section{Crush injury model generation}

For inducing the muscle acute injury we adapted a previously described crush injury model $[36,37]$ with minor changes. Thirty-nine female C57BL/6 8-week-old mice (Envigo, Netherlands) were administered with buprenorphine (Temgesic ${ }^{\circ}$, Reckitt Benckiser, Switzerland) prior to surgery, followed by anesthesia with $2 \%$ isoflurane (Forene $^{\mathrm{rm}}$, Abbvie) exhalation. Both lower limbs were immobilized, and a $1.5-\mathrm{cm}$ longitudinal incision of the skin was made, exposing the tibialis anterior (TA) muscle. The TA was separated from the tibia and underlying extensor digitorum longus (EDL) muscle. The crush injury was induced on both left and right TA muscles via surgical clamps (Fine Instruments, Germany). The muscles were manually clamped twice over their complete lengths. Immediately after the injury, the ADSC mixed with the collagen hydrogel (Rat collagen I; Corning), or collagen only as control, were implanted into randomly assigned crushed muscle. For each mouse, one TA was always implanted with ADSC and the other TA from the same animal served as a collagen-treated control. To track the ADSC after implantation, the cells were labeled prior to implantation with $\mathrm{PKH} 26$-red fluorescent linker (Sigma-Aldrich) according to the manufacturer's recommendations. For optical projection tomography (OPT) analysis, the ADSC were labeled with Cell Tracker Orange CMTMR $^{\mathrm{m} \mathrm{m}}$ dye (Thermo Fisher Scientific) according to the manufacturer's recommendations. A total of $3 \times 10^{6}$ ADSC in suspension in $50 \mu \mathrm{l}$ collagen hydrogel were implanted.

Morphological, histological, and functional data were collected at 7,14 , and 28 days postinjury. The contractility of the TA muscles was measured ex vivo in an organ bath system and healthy age- and sex-matched animals served as controls.

\section{Functional measurements of TA muscles}

The isometric contractile measurements were performed with the Radnoti Organ Bath System (AD Instruments) coupled with a square pulse electrical stimulator (Grass model S88) and data acquisition platform (AD instruments Power Lab Data Acquisition System and Lab Chart software, Oxford, UK) as described previously [38]. Briefly, the TA muscles were dissected and fixed in the organ bath chamber filled with $5 \mathrm{ml}$ Krebs Henseleit (Sigma-Aldrich) solution at $25{ }^{\circ} \mathrm{C}$ and oxygenated with $\mathrm{O}_{2} / \mathrm{CO}_{2}(95 \% / 5 \%)$. For a single twitch tension measurement, the muscle was stimulated with a single $0.5-\mathrm{ms}$ square pulse at $40 \mathrm{~Hz}$. The tetanus tension frequency was established from the force-frequency relationship, where the maximal force for the healthy muscles is achieved at $120 \mathrm{~Hz}$. The tetanus tension was induced by stimulation with a pulse train for $300 \mathrm{~ms}$ at $40 \mathrm{~V} \mathrm{~Hz}$. The muscles were rested for $3 \mathrm{~min}$ between each series and all the force measurements were normalized to the weight of each individual muscle.

\section{Analysis of TA muscle regeneration Immunohistochemistry}

TA muscles were harvested, snap frozen, and cryosectioned at $7 \mu \mathrm{m}$. Sections were fixed with $4 \%$ paraformaldehyde, permabilized with $0.1 \%$ Triton-X, and blocked with 5\% BSA for $1 \mathrm{~h}$. Primary antibody $\alpha$-actinin (Sigma-Aldrich) was detected using the M.O.M kit (Dako, Glostrup, Denmark). The CD31 (Abcam) and Ki67 (Dako) antibodies after overnight incubation at $4{ }^{\circ} \mathrm{C}$ were detected using goat anti-rabbit or donkey anti-goat IgG antibodies conjugated to FITC.

\section{Histological analysis}

Cryosections were fixed with $4 \%$ paraformaldehyde and stained for hematoxylin and eosin using standard procedures. The cross-sections were visualized using a Pannoramic 250 Flash III slide scanner (Sysmex Suisse AG, Horgen, Switzerland) and images were acquired using Pannoramic Control Software (3D Histotech, Budapest, Hungary). Myofibers diameters were determined using a Pannoramic Viewer (3D Histotech).

\section{Optical projection tomography}

To track ADSC incorporation within the whole TA muscle without physical sectioning we used OPT. The TA muscles were harvested from 16 female C57BL/6 8-week-old mice (Envigo, Netherlands) and embedded in $2 \%$ ultrapure low-gelling agarose (Sigma). Samples were dehydrated in 100\% methanol (Sigma) overnight at room temperature and then cleared in a BABB solution (benzyl 
alcohol and benzyl benzoate) for a minimum of $3 \mathrm{~h}$ at room temperature. The optical sections were acquired with Optical Projection Tomography Scanner (Bioptonics) and analyzed with Imaris software (BitPlane, Zurich, Switzerland).

\section{Protein isolation and Western blotting}

Total protein extracts were isolated from cells using RIPA buffer supplemented with protease inhibitor cocktail (P8340 Sigma-Aldrich). The protein concentration was determined with the BCA protein assay (Thermo Fisher Scientific, Ecublens, Switzerland).

A total of $20 \mu \mathrm{g}$ protein from each sample was separated by SDS-PAGE and transferred to polyvinylidene difluoride (PVDF) membranes (Trans-Blot Turbo, Bio-Rad Laboratories, Cressier, Switzerland). The primary antibodies used were: alpha-actinin (Cell Signaling Technology, Danvers, USA), MyHC (DSHB), MyoD (Santa Cruz Biotechnology), Pax7 (Thermo Fisher), and GAPDH (Cell Signaling). Primary antibodies were detected using anti-rabbit or anti-mouse horseradish peroxidase (HRP)-conjugated IgG (DAKO). Signals were visualized using enhanced chemiluminescence (Western Lightning Plus ECL, Perkin Elmer) and developed with CURIX 60 (AGFA). Quantification of the signal was performed using ImageJ software and all the signals were normalized to glyceraldehyde-3-phosphate dehydrogenase (GAPDH) expression.

\section{$R T-q P C R$}

Total RNA was isolated from all mouse samples and cells using the TRIzol reagent-based method following the manufacturer's protocol (Life Technologies). RNA was reverse-transcribed with the Omniscript RT Kit 200 (Qiagen). RT-qPCR was performed using the ABI PRISM 7900 Sequence Detection System (Applied Biosystems) according to the standard protocols. The following mouse TaqMan (Life Technologies) probes were used: Pax7 (Mn01354484_m1), MyoD (Mm00440387_m1), MyHC (Mm00443013_m1), collagen type I (Coll1; Mm0048388 8_m1), transforming growth factor (TGF)b1 (Mm011788 20_m1), and GAPDH (Mm99999915_g1). Expression levels were normalized to the expression level of the GAPDH housekeeping gene. The experiment was repeated with at least three biological replicates and each sample was analyzed in triplicate.

\section{Statistical analysis}

Statistical tests were performed using GraphPad Prism 6 software (GraphPad Prism Software Inc.). Student's $t$ tests were performed for RT-qPCR analysis and WB quantification. For the organ bath analysis, one-way analysis of variance (ANOVA) with Bonferroni correction and paired $t$ test were performed. For the histological analysis of the fiber size distribution, two-way ANOVA with multiple comparisons and Sidak corrections were performed. $p<0.05$ was considered statistically significant. Error bars represent the mean \pm standard deviation (SD).

\section{Results}

\section{Characterization of mouse ADSC and SC}

ADSC were isolated and cultured until passage 3 as a morphologically homogenous population (Additional file 1: Figure S1A). Greater than $95 \%$ of the population expressed the MSC-specific surface markers CD29, CD44, and CD90, and were negative for hematopoietic stem cell CD34 and endothelial surface marker CD31 (Additional file 1: Figure S1B). Immunocytochemistry confirmed the surface expression of CD29, CD44, and CD90 markers, as well as negative expression for CD31 (Additional file 1: Figure S1C). Expression of all markers was consistent throughout the in-vitro and in-vivo experiments. The primary mouse SC cultured until passage 3 in the high-serum proliferation medium exhibited a uniform, undifferentiated morphology (Additional file 2: Figure S2A) and were positive for Pax7 transcription factor (Additional file 2: Figure S2B). The myogenic potential of the isolated SC was confirmed by transferring the cells into differentiation medium, where a subset of cells upregulated the MyoD transcription factor (Additional file 2: Figure S2C) and further formed multinucleated myotubes, positive for the skeletal muscle-specific structural protein $\mathrm{MyHC}$ (Additional file 2: Figure S2D-F).

\section{Effect of ADSC on satellite cell activation and differentiation in vitro}

To investigate the stimulatory effect of ADSC on SC in vitro, we compared the expression level of myogenic markers between SC directly cocultured with ADSC versus $\mathrm{SC}$ cultured in the conditioned medium derived from the ADSC (CM-ADSC). SC directly cocultured with ADSC in proliferative medium after $72 \mathrm{~h}$ showed increased mRNA and protein expression of the transcription factor Pax7 (Fig. 1a, b). For SC cultured in proliferative CM-ADSC the same stimulating effect on Pax7 expression was not observed (Fig. 1c). Additionally, in proliferative medium under both culture conditions there were no differences in MyoD expression (Fig. 1a-c). $\mathrm{SC}$ cultured with ADSC in differentiation-induction medium also did not show upregulation of $\mathrm{MyoD}$ or $\mathrm{MyHC}$ expression in both experimental setups of direct coculture and CM-ADSC (Fig. 1d-f). Moreover, cells cultured in CM-ADSC demonstrated a decreased level of MyHC mRNA expression (Fig. 1f). Additionally, for both experimental groups cultured in differentiation-induction medium, the distribution of the average number of nuclei per myotube was similar (Fig. 1g). 


\section{ADSC improve skeletal muscle contractility}

Immediately following a crush injury to the TA muscle, ADSC were implanted and functional analysis in the organ bath system was performed at days 7, 14, and 28 postinjury (Fig. 2a). From the functional analysis, we observed a significant increase in contractility for twitch and tetanus tension at 28 days for the ADSC-treated muscles in comparison with the collagen-treated TAs and muscles that did not receive any treatment (Fig. 2b). Additionally, the strength of the ADSC-treated muscles was similar to the healthy TAs from the healthy age-matched mice. After pairing the muscles from the same animals, ADSC-treated muscles performed better than the collagen controls (Fig. 2c). Moreover, we did not observe any differences in the average muscle weight between the collagen- and cell-treated samples at any of the time points; however, a significant muscle weight reduction in the untreated group was observed at 14 and 28 days postinjury (Fig. 2 d).

\section{ADSC engraft into damaged tissue but do not contribute to skeletal muscle formation in vivo}

To elucidate the mechanisms underlying the enhanced contractility of the ADSC-treated muscles, we tracked the implanted cells with OPT microscopy which allowed us to visualize the three-dimensional (3D) pattern of cell distribution within the whole muscle (Additional file 3: Movie S1, Additional file 4: Movie S2, Additional file 5: Movie S3, Additional file 6: Movie S4). At 7 days postinjury, we observed that implanted cells colocalize with the site of the damage, corresponding to $30 \%$ of the whole TA volume (Fig. 3c, Additional file 4: Movie S2). At the subsequent time points, we observed a reduction in the number of implanted cells, as well as a decrease in the injury size (Additional file 5: Movie S3, Additional file 6: Movie S4; Additional file 7: Figure S4).

We also detected a fraction of ADSC aligned along healthy myofibers at the border of the injury (Fig. 3a, lower panel). No donor cells were found to be differentiated into myofibers, fused with existing myotubes, or
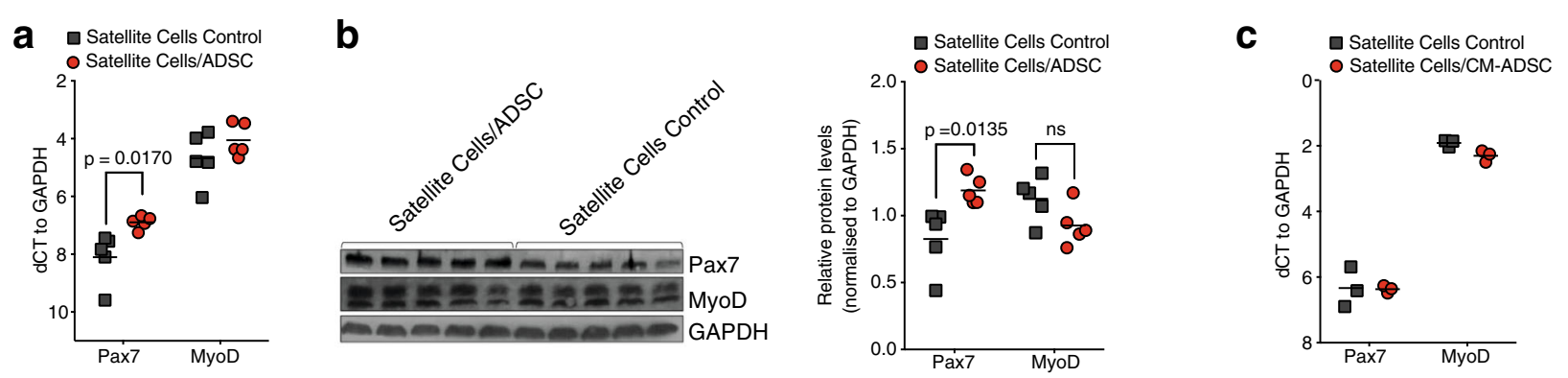

d
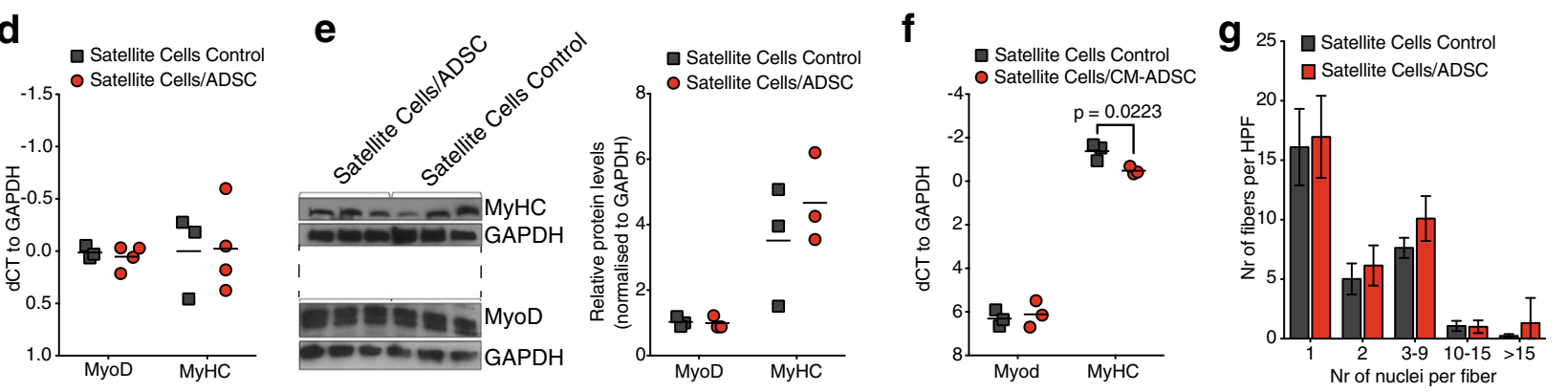

Fig. 1 Effect of adipose-derived stem cells (ADSC) on satellite cell (SC) activation and differentiation. a Relative mRNA level of paired box protein domain 7 (Pax7) and myogenic differentiation antigen (MyoD) transcription factors in SC cocultured with/without ADSC in proliferation-induction medium. b Protein expression of Pax7 and MyoD (upper band) transcription factors in SC cocultured with/without ADSC in proliferation-induction medium. c Relative mRNA level of Pax7 and MyoD expressed in SC in proliferation-induction ADSC conditioned medium (CM-ADSC). d Relative mRNA level of MyoD and MyHC in MPC cocultured in differentiation-inducing medium with/ without ADSC. e Relative protein expression of MyoD and MyHC in MPC in differentiation medium cocultured with/without ADSC and relative mRNA levels of expression of $M y O D$ and $M y H C$ in a differentiation-inducing conditioned medium from ADSC. $\mathbf{f}$ Relative mRNA levels of MyoD and MyHC expressed in MPC in differentiation-induction CM-ADSC. g Average number of multinucleated fibers per highpower filed (HPF) between MPC cocultured with/without ADSC, shown as mean \pm SD. The relative level of mRNA was measured using RTqPCR and the relative protein level was determined with Western blot. All the results were normalized to glyceraldehyde-3-phosphate dehydrogenase (GAPDH) expression 


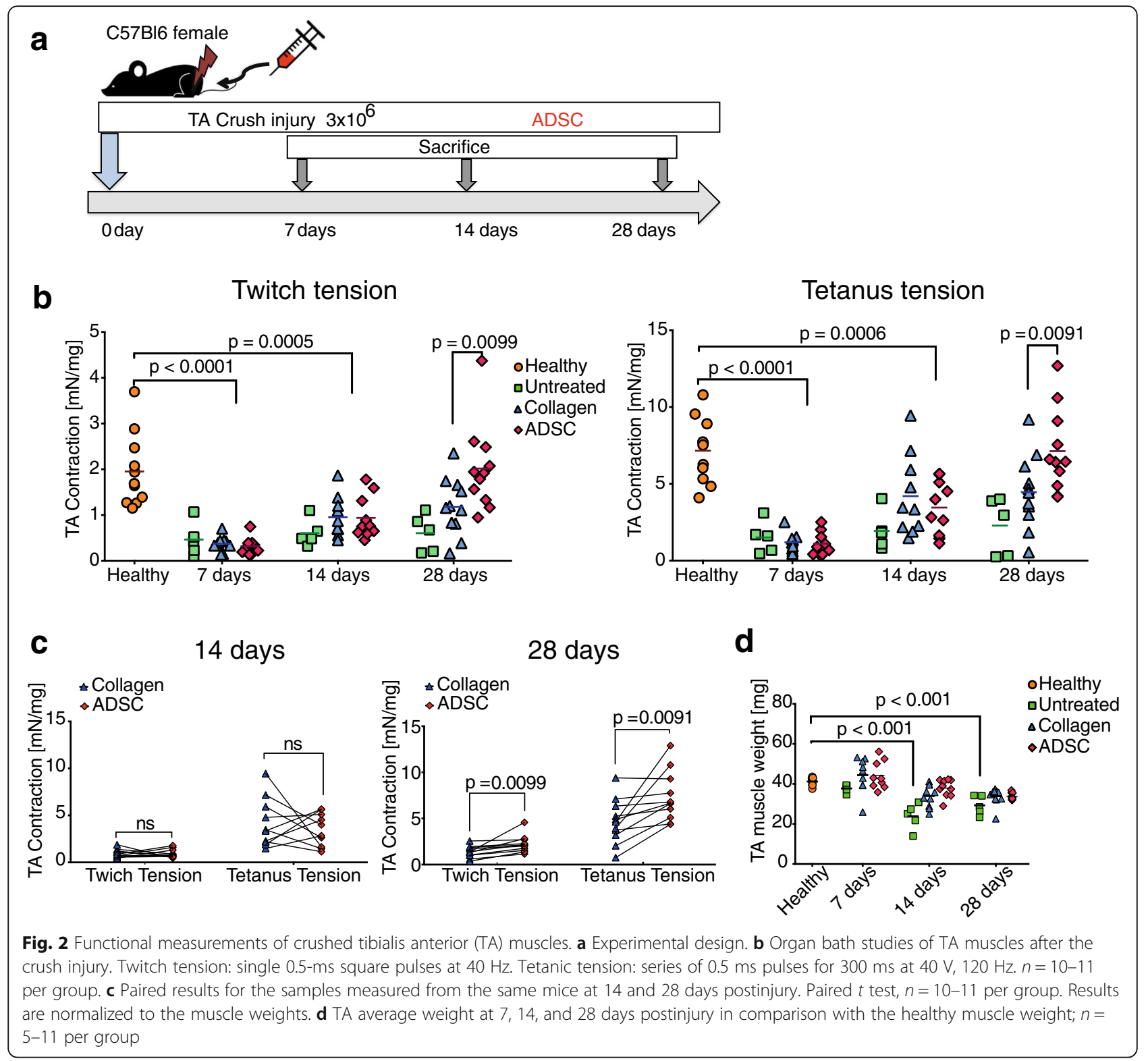

positive for the proliferation marker Ki67 (Fig. 3b) or endothelial marker CD31 (Additional file 8: Figure S3).

\section{ADSC treatment accelerates myoblast expansion}

Seven days after the crush injury, most of the damaged area was occupied by mononucleated cells, and sporadic myofibers were present at the periphery of the muscle (Fig. 4). By 14 days postinjury, we observed a higher accumulation of myofibers and a smaller diameter in ADSC-treated muscles. Additionally, we detected a significantly higher number of centrally nucleated immature myofibers in ADSC-treated muscles when compared with the collagen-treated control (Fig. 4b).

At 28 days postinjury, the fraction of large myofibers in the ADSC-treated muscles was significantly higher than in the control samples and, at the same time, the small myofibers were dominant in the control samples. In both experimental groups no fibrotic tissue at 28 days following damage was observed.

\section{ADSC do not impact myogenesis in early regenerative stages}

Pax7 was upregulated immediately upon injury and peaked between days 5 and 7 following damage (Fig. 5a). This was accompanied by immediate upregulation of MyoD during the first week postinjury, which points to the high myogenesis. The $\mathrm{MyHC}$ was downregulated within this time and was restored at later time points. In our study, we did not observe any differences in the expression of these early or late myogenesis markers 

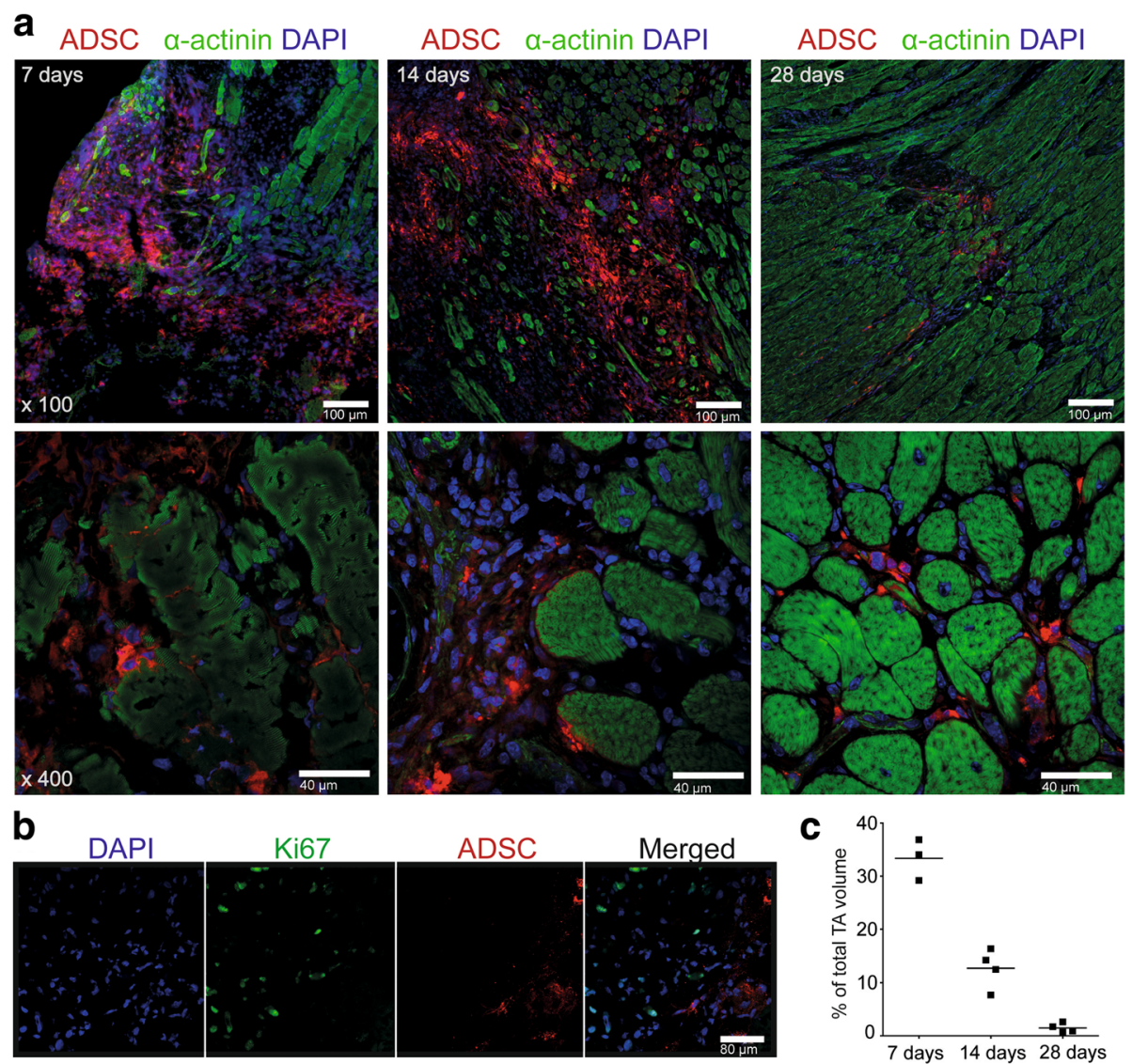

Fig. 3 Incorporation of the implanted adipose-derived stem cells (ADSC) into crushed tibialis anterior (TA) muscles. a Tracing of the PKH26 red fluorescently labeled ADSC after 7, 14, and 28 days following cell transplantation. Frozen sections of TA muscle were counterstained for a-actinin (green) and cell nuclei (DAPI, blue). Upper panel: scale bars $=100 \mu \mathrm{m}$; bottom panel: scale bars $=40 \mu \mathrm{m}$. b Ki67 staining showing that fluorescently red-labeled ADSC do not proliferate after implantation. Scale bar $=40 \mu \mathrm{m}$. c Quantification of the ADSC reduction within the TA muscles over time; $n=3-4$ per group

between the ADSC-treated muscles and collagen-treated controls.

The main component of the muscle extracellular matrix (ECM), collagen type I (Coll1) was upregulated during the entire regenerative process; however, 7 days postinjury we observed higher collagen type I mRNA levels in the collagen-treated controls. This observation did not correlate with TGF 31 expression (Fig. 5b).

\section{Discussion}

Developing an autologous stem cell therapy for large skeletal muscle injuries would be of great benefit for patients suffering from muscle function loss due to large acute injuries. In regenerative medicine, ADSC are an attractive source of easily accessible MSC with a great regenerative potential. In this study, we demonstrated that a single transplantation of ADSC into the crushed tibialis anterior immediately after the injury significantly accelerates muscle repair in mice by increasing myofiber diameter as well as higher twitch and tetanic force generation. ADSC-treated muscles also showed an earlier appearance of centrally nucleated myofibers, which could explain the greater force generation. Although the possibility of ADSC myogenic transdifferentiation in vivo has been reported $[29,39]$, in our study we did not observe direct participation of the implanted ADSC in new myofiber formation. This result points towards a possible paracrine modulatory effect of the implanted cells, which would be in line with the growing number of reports describing the supportive properties of ADSC [40-43]. Unfortunately, we were not able to determine the mechanism by which the ADSC are cleared from the injured areas. ADSC could share a similar fate as muscle-resident fibro-adipogenic progenitors (FAPs). It was recently shown that FAPs are activated upon injury to support the first phase of muscle regeneration by paracrine signaling. Once the inflammatory phase of the muscle regeneration is finished, FAPs undergo apoptosis [44-46]. 


\section{a}

7 days ADSC

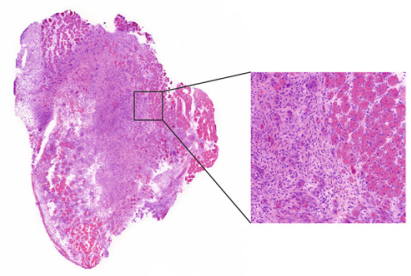

14 days ADSC

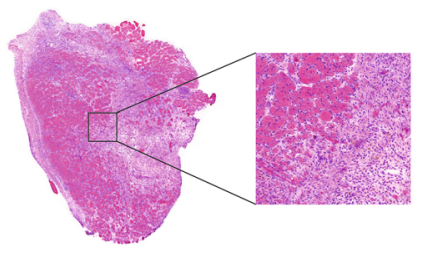

28 days ADSC

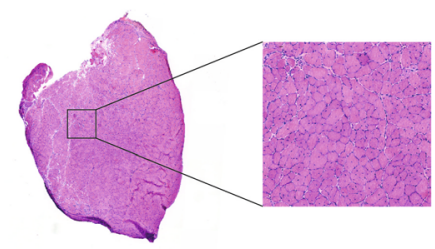

b
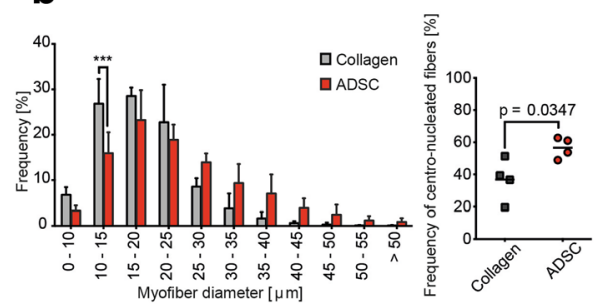
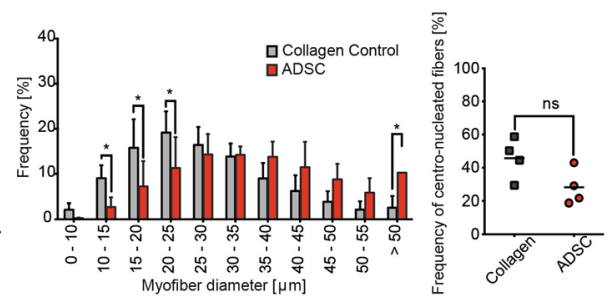

Fig. 4 Adipose-derived stem cell (ADSC) treatment positively affects skeletal muscle regeneration. a Representative cross sections of TA muscles at 7, 14, and 28 days following crush injury with ADSC or collagen transplantation. b Frequency of fiber size distribution for defined ranges of cross-sectional diameter in TA at 14 and 28 days following crush injury with ADSC or collagen transplantation and frequency of centrally nucleated fibers at 14 and 28 days postinjury. Results are shown as mean \pm SD from $n=3-5$ experimental repeats. ${ }^{*} p<0.05$, ${ }^{* * *} p<0.001$

We demonstrated that ADSC have a positive stimulatory effect on SC activation in vitro. Direct coculturing with ADSC induces Pax7 expression in SC when compared with conditioned medium. Therefore, the cross-talk between these two cell populations seems to be essential for SC activation. Additionally, we found that culturing $\mathrm{SC}$ in CM-ADSC leads to MyHC downregulation. This observation supports the use of stem cell implantation into the injury site, where the ADSC are expected to have a higher impact on muscle regeneration than delivering only ADSC conditioned medium. This ex-vivo finding is inconsistent with in-vivo studies, where it has been shown that injection of the supernatant is sufficient for achieving a muscle regeneration level comparable to ADSC implantation [47, 48].
From the functional analysis of the harvested muscles, we found that intramuscular transplantation of ADSC into acute injured tibialis anterior muscle helps to restore its contractility within 28 days. There was no indication that the implanted ADSC underwent myogenic or endothelial differentiation, thus suggesting a supportive role of ADSC via paracrine and cell-cell mechanisms. These observations are in line with previous reports, where the implantations of the ADSC into injured muscles have shown a positive effect [49-52]. Additionally, reduction in the weight of the untreated muscles together with very poor functional improvement shows the potential beneficial effect of the collagen hydrogel itself. 


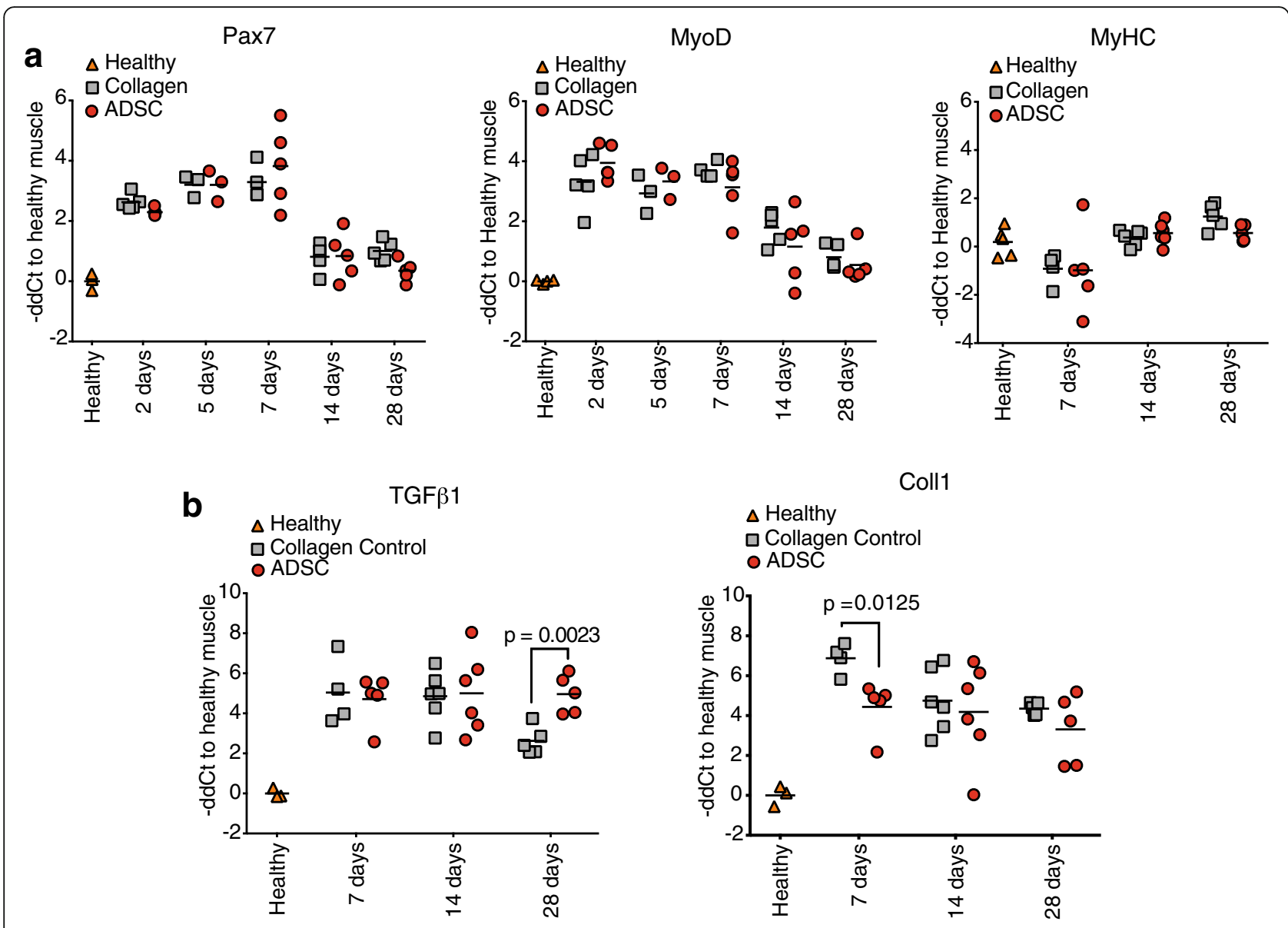

Fig. 5 Effect of adipose-derived stem cell (ADSC) transplantation on myogenesis and fibrosis in regenerating TA. a Comparative analysis of mRNA from crushed TAs treated with ADSC or collagen hydrogel only. RT-qPCR analysis showed the expression levels of the transcription factors paired box protein domain 7 (Pax7) and myogenic differentiation antigen (MyoD) and structural component myosin heavy chain (MyHC) over time. b Comparative level of mRNA measured by RT-qPCR of collagen type I (Coll1) and transforming growth factor $\beta 1$ (TGF $\beta 1$ ). All results are normalized to GAPDH expression and plotted in relation to healthy TA expression level; $n=3-5$ per group

One of the major causes of impaired muscle functionality after injury is permanent fibrotic tissue formation. However, in this animal model we did not see connective tissue deposition at the 28-day time point in either experimental groups. This excludes the fibrosis formation as an explanation of the functional discrepancy between the ADSC-treated muscles and the collagen controls. Moreover, the elevated TGF $\beta 1$ expression in the ADSC-treated muscles observed at 28 days postinjury was not caused by scar tissue formation. In several studies, TGF $\beta 1$ has been described as the most important factor responsible for persistent fibrosis in skeletal muscles [53], but mostly in chronic conditions $[54,55]$. In our study, the upregulation of the TGF $\beta 1$ could be than associated with the further ECM remodeling; however, the lack of the elevated collagen 1 expression at 28 days postinjury makes this highly unlikely.

Based on the histological assessment of the cross-sectional areas, we concluded that the significant higher force produced in the ADSC-treated group is an effect of a more efficient remodeling and muscle regeneration. It has been proven that the number and the size of the myofibers directly correlates with the strength of the muscle [56], and in our study the frequency distribution for the myofibers size is shifted towards higher number of large and centrally nucleated immature myofibers in the ADSC-treated muscles. The work of Pecanha et al. suggests a similar mechanism, where an injection of ADSC into lacerated soleus rat muscle results in increased force production at 2 weeks postinjury but not at 4 weeks, suggesting that ADSC treatment accelerates muscle recovery [51]. This also implies that we cannot exclude the possibility of the full recovery of the collagen-treated muscles at later time points.

In our in-vivo experimental setup, we did not observe any differences in Pax7 expression between the ADSC-treated muscles and collagen controls. This could be due to the animal model itself, where the crush injury 
triggers a very high level of SC activation. Other possible explanations are that there is an attenuated effect of the ADSC on SC activation in vivo, or a lack of specificity in our analysis. In our experiment, the RNA was isolated from the site of injury. However, the crush injury activates not only the SC in the injured area, but also SC along the whole myofiber, which might have biased our results.

The last possibility explaining the faster functional recovery of the ADSC-treated muscles is their possible impact on vasculogenesis. Immature myoblasts can function and differentiate in the hypoxic condition that is dominating at the initial phase of the muscle injury. However, for the myoblast fusion and myotube maturation the regrowth of the vasculature is essential [57]. It has been proven in several studies that ADSC improve the vasculature growth and new vessel formation either by differentiation into endothelial cells or by secreting vascular endothelial growth factor (VEGF) [31, 42, 58]. In our crush injury model we did not observe ADSC differentiation into endothelial cells; however, based on the recent published data showing the MSC-mediated vasculature repair of the damaged tissues correlating with increased VEGF levels, we cannot exclude the hypothesis that implanted ADSC support in-vivo muscle regeneration by proangiogenic factor secretion [59-61]. However, this hypothesis needs to be further verified.

\section{Conclusions}

In the present study, we showed that ADSC therapy accelerates the functional recovery of skeletal muscle in mice. This was shown by a significant increase in both twitch and tetanic force and an increase in the number of large, regenerating myofibers. We did not detect any ADSC myogenic differentiation, and therefore we suggest that ADSC act via direct cell-cell or paracrine mechanisms by secreting factors involved in transient acceleration of skeletal muscle repair. Summarizing, ADSC remain attractive candidates for skeletal muscle regeneration therapies; however, further studies are necessary to fully understand their mechanisms of action.

\section{Additional files}

Additional file 1: Figure S1. Characterization of mouse primary ADSC. (A) Isolated ADSC at passage 3 present characteristic fibroblast-like morphology. (B) Gating strategy for flow cytometry analysis. (C) Flow cytometry analysis shows that ADSC were positive for the MSC surface markers CD44 (97\% \pm 2.2$), C D 90(95.5 \% \pm 1.5), C D 29(98.6 \% \pm 0.7)$, and PDGFRB $(57.3 \% \pm 8.8)$, and negative for the hematopoietic stem cell surface marker CD34 $(0.8 \% \pm 0.2)$ and endothelial surface marker CD31 $(1.2 \% \pm 0.4)$. Data from three independent experiments as mean $\pm S D$. (D) Representative immunocytochemistry confirms the expression of the selected surface markers on the ADSC. (JPG $2826 \mathrm{~kb}$ )

Additional file 2: Figure $\mathbf{S 2}$. Characterization of mouse primary satellite cells. (A) Undifferentiated SC at 60\% confluency at passage 3. (B) SC are positive for Pax7 transcription factor. (C) MPC are a fraction of SC committed to myogenesis expressing MyoD transcription factor. (D) Multinucleated myotubes (black arrows) formed by fusion of SC (white arrows) at 7 days in differentiation culture conditions. (E) Fiber formation assay demonstrating long, multinucleated myotubes. Giemsa staining at 5 days in differentiation medium. (F) Myotubes express skeletal musclespecific myosin heavy chain (MyHC). (JPG 3594 kb)

Additional file 3: Movie S1. Control uninjured TA. Optical projection tomography single plane of crushed TAs with implanted ADSC. Blue: myofibers. Red: implanted ADSC. (MP4 $387 \mathrm{~kb}$ )

Additional file 4: Movie S2. TA crush injury 7 days. Optical projection tomography single plane of crushed TAs with implanted ADSC. Blue: myofibers. Red: implanted ADSC. (MP4 700 kb)

Additional file 5: Movie S3. TA crush injury 14 days. Optical projection tomography single plane of crushed TAs with implanted ADSC. Blue: myofibers. Red: implanted ADSC. (MP4 $470 \mathrm{~kb}$ )

Additional file 6: Movie S4. TA crush injury 28 days. Optical projection tomography single plane of crushed TAs with implanted ADSC. Blue: myofibers. Red: implanted ADSC. (MP4 382 kb)

Additional file 7: Figure S4. OPT of single plane projection of the crushed TAs with implanted ADSC and collagen treated controls at 7, 14 and 28 days postimplantation. Blue: myofibers. Red: implanted ADSC. (JPG $2385 \mathrm{~kb}$ )

Additional file 8: Figure S3. ADSC do not differentiate into endothelial cells. Representative CD31 (green) staining showing that fluorescently red-labeled ADSC do not overlap with the endothelial cells in the TA muscle. Frozen sections of TA muscle were counterstained for cell nuclei (DAPI, blue). (JPG $5130 \mathrm{~kb}$ )

\section{Abbreviations}

ADSC: Adipose-derived stem cells; ANOVA: Analysis of variance; BSA: Bovine serum albumin; CEE: Chicken embryonic extract; CM: Conditioned medium; Coll1 : Collagen type 1; DMEM: Dulbecco's modified Eagle's medium; ECM : Extracellular matrix; EDL : Extensor digitorum longus; FAP: Fibro-adipogenic progenitor; FBS: Fetal bovine serum; FITC: Fluorescein isothiocyanate; GAPDH: Glyceraldehyde-3-phosphate dehydrogenase; HS: Horse serum; MPC: Muscle precursor cells; MSC: Mesenchymal stem cells; MyHC : Myosin heavy chain; MyoD : Myogenic differentiation antigen; OPT : Optical projection tomography; Pax7 : Paired box protein domain 7; PS: Penicillinstreptomycin; RT-qPCR: Real-time quantitative polymerase chain reaction; Sca-1 : Stem cell antigen 1; SC: Satellite cells; SD: Standard deviation; TA: Tibialis anterior; TGF: Transforming growth factor; VEGF: Vascular endothelial growth factor; WB: Western blot

\section{Acknowledgements}

We thank Nathalie Rion for help with satellite cell isolation, Fabian Blank, the MIC and the LCI Core Facility, for support with the LSM 710 confocal microscope at the University of Bern, and Stefan Müller from the FACS Lab core facility at the University of Bern for support with the flow cytometry analysis.

\section{Funding}

This study was supported by grants from Ruth \& Arthur Scherbarth Stiftung, Switzerland.

\section{Availability of data and materials}

The datasets used and/or analyzed during the current study are available from the corresponding author on reasonable request.

\section{Authors' contributions}

AG carried out all the experiments, participated in the design of the study, and drafted the manuscript. SS participated in designing the study, helped in acquisition of the data, participated in the interpretation and analyzing of the results, and critically revised the manuscript. DH participated in designing the in-vivo study, and interpretation and analysis of the data. FM participated in designing the OPT experiment, acquired and helped in analyzing the OPT data. DS was involved in data interpretation and revising the manuscript. DC participated in the design of the study and manuscript revision. DE was involved in the design of the study, interpretation of data, and 
revising the manuscript. LB participated in the design of the study, interpretation of data, and critical revision of the manuscript. All authors read and approved the manuscript.

\section{Ethics approval}

All procedures performed in this study involving animals were in accordance with the ethical standards of the Federation for Laboratory Animal Science Associations guidelines and approved by the Animal Care Committee of the Canton Bern, Switzerland.

\section{Consent for publication}

Not applicable.

\section{Competing interests}

The authors declare that they have no competing interests.

\section{Publisher's Note}

Springer Nature remains neutral with regard to jurisdictional claims in published maps and institutional affiliations.

\section{Author details}

'Department of Clinical Research, Laboratory for Visceral Surgery and Medicine, University of Bern, Murtenstrasse 35, 3008 Bern, Switzerland. ${ }^{2}$ Department of Urology, Laboratory for Tissue Engineering and Stem Cell Therapy, University Hospital Zurich, Zurich, Switzerland. ${ }^{3}$ University Clinic for Visceral Surgery and Medicine, Bauchzentrum Bern, Inselspital, CH-3010 Bern, Switzerland. ${ }^{4}$ Theodor Kocher Institute, University of Bern, Bern, Switzerland. ${ }^{5}$ Current address: Division of Immunology, Transplantation and Infectious Diseases, IRCCS San Raffaele Scientific Institute, Milan, Italy.

\section{Received: 17 November 2017 Revised: 29 May 2018}

Accepted: 8 June 2018 Published online: 17 July 2018

\section{References}

1. Ten Broek RW, Grefte S, Von den Hoff JW. Regulatory factors and cell populations involved in skeletal muscle regeneration. J Cell Physiol. 2010:224(1):7-16.

2. Huard J, Li Y, Fu FH. Muscle injuries and repair: current trends in research. J Bone Joint Surg Am. 2002;84-A(5):822-32.

3. Lieber RL, Friden J. Functional and clinical significance of skeletal muscle architecture. Muscle Nerve. 2000;23(11):1647-66

4. Sambasivan $\mathrm{R}$, et al. Pax7-expressing satellite cells are indispensable for adult skeletal muscle regeneration. Development. 2011;138(17):3647-56.

5. Lepper C, Partridge TA, Fan CM. An absolute requirement for Pax7-positive satellite cells in acute injury-induced skeletal muscle regeneration. Development. 2011:138(17):3639-46.

6. Rudnicki MA, et al. The molecular regulation of muscle stem cell function. Cold Spring Harb Symp Quant Biol. 2008;73:323-31.

7. von Maltzahn J, et al. Pax7 is critical for the normal function of satellite cells in adult skeletal muscle. Proc Natl Acad Sci U S A. 2013;110(41):16474-9.

8. Collins CA, et al. Stem cell function, self-renewal, and behavioral heterogeneity of cells from the adult muscle satellite cell niche. Cell. 2005; 122(2):289-301.

9. Hawke TJ, Garry DJ. Myogenic satellite cells: physiology to molecular biology. J Appl Physiol (1985). 2001;91(2):534-51.

10. Grogan BF, Hsu JR. Volumetric muscle loss. J Am Acad Orthop Surg. 2011; 19(Suppl 1):S35-7.

11. Jarvinen TA, Jarvinen $M$, Kalimo $H$. Regeneration of injured skeletal muscle after the injury. Muscles Ligaments Tendons J. 2013:3(4):337-45.

12. Gates C, Huard J. Management of skeletal muscle injuries in military personnel. Operative Techniques in Sports Medicine. 2005;13(4):247-56.

13. Lin $\mathrm{CH}$, et al. Free functioning muscle transfer for lower extremity posttraumatic composite structure and functional defect. Plast Reconstr Surg. 2007;119(7):2118-26.

14. Burns TC, et al. Does the zone of injury in combat-related type III open tibia fractures preclude the use of local soft tissue coverage? J Orthop Trauma. 2010;24(11):697-703

15. Sacco A, et al. Self-renewal and expansion of single transplanted muscle stem cells. Nature. 2008;456(7221):502-6.

16. Cerletti $M$, et al. Highly efficient, functional engraftment of skeletal muscle stem cells in dystrophic muscles. Cell. 2008;134(1):37-47.
17. Tedesco FS, et al. Repairing skeletal muscle: regenerative potential of skeletal muscle stem cells. J Clin Investig. 2010;120(1):11-9.

18. Sampaolesi $M$, et al. Mesoangioblast stem cells ameliorate muscle function in dystrophic dogs (vol 444, pg 574, 2006). Nature. 2014;507(7491):262.

19. Zuk PA, et al. Multilineage cells from human adipose tissue: implications for cell-based therapies. Tissue Eng. 2001;7(2):211-28.

20. El Atat $\mathrm{O}$, et al. An evaluation of the stemness, paracrine, and tumorigenic characteristics of highly expanded, minimally passaged adipose-derived stem cells. PLoS One. 2016;11(9):e0162332.

21. Tang W, et al. White fat progenitor cells reside in the adipose vasculature. Science. 2008:322(5901):583-6.

22. Housman TS, et al. The safety of liposuction: results of a national survey. Dermatol Surg. 2002;28(11):971-8.

23. Mizuno $\mathrm{H}$, et al. Myogenic differentiation by human processed lipoaspirate cells. Plast Reconstr Surg. 2002:109(1):199-209. discussion 210-1.

24. Zhang $L$, et al. Chondrogenic differentiation of human mesenchymal stem cells: a comparison between micromass and pellet culture systems. Biotechnol Lett. 2010;32(9):1339-46.

25. Cardozo AJ, Gomez DE, Argibay PF. Neurogenic differentiation of human adipose-derived stem cells: relevance of different signaling molecules, transcription factors, and key marker genes. Gene. 2012;511(2):427-36.

26. Ning $\mathrm{H}$, et al. Conversion of adipose-derived stem cells into natural killer-like cells with anti-tumor activities in nude mice. PLoS One. 2014;9(8):e106246.

27. Gwak SJ, et al. In vitro cardiomyogenic differentiation of adipose-derived stromal cells using transforming growth factor-beta1. Cell Biochem Funct. 2009;27(3):148-54

28. Izadpanah $\mathrm{R}$, et al. Biologic properties of mesenchymal stem cells derived from bone marrow and adipose tissue. J Cell Biochem. 2006:99(5):1285-97.

29. Di Rocco G, et al. Myogenic potential of adipose-tissue-derived cells. J Cell Sci. 2006:119(Pt 14):2945-52.

30. Bayati $V$, et al. The evaluation of cyclic uniaxial strain on myogenic differentiation of adipose-derived stem cells. Tissue Cell. 2011:43(6):359-66.

31. Strem BM, et al. Multipotential differentiation of adipose tissue-derived stem cells. Keio J Med. 2005:54(3):132-41.

32. Yong KW, et al. Paracrine effects of adipose-derived stem cells on matrix stiffness-induced cardiac myofibroblast differentiation via angiotensin II type 1 receptor and Smad7. Sci Rep. 2016;6:33067.

33. Sowa $Y$, et al. Adipose-derived stem cells produce factors enhancing peripheral nerve regeneration: influence of age and anatomic site of origin. Stem Cells Dev. 2012;21(11):1852-62.

34. Bunnell BA, et al. Adipose-derived stem cells: isolation, expansion and differentiation. Methods. 2008:45(2):115-20.

35. Pasut $A$, Jones $A E$, Rudnicki MA. Isolation and culture of individual myofibers and their satellite cells from adult skeletal muscle. J Vis Exp. 2013;73:e50074

36. Stratos I, et al. Vitamin D increases cellular turnover and functionally restores the skeletal muscle after crush injury in rats. Am J Pathol. 2013;182(3):895-904.

37. Matziolis $G$, et al. Autologous bone marrow-derived cells enhance muscle strength following skeletal muscle crush injury in rats. Tissue Eng. 2006; 12(2):361-7.

38. Oishi PE, Cholsiripunlert S, Gong W, Baker AJ, Bernstein HS. Myo-mechanical analysis of isolated skeletal muscle. J Vis Exp 2011;(48):2582. https://doi.org/ $10.3791 / 2582$

39. Zhang $Y$, et al. Long-term engraftment of myogenic progenitors from adipose-derived stem cells and muscle regeneration in dystrophic mice. Hum Mol Genet. 2015:24(21):6029-40.

40. Salgado AJ, et al. Adipose tissue derived stem cells secretome: soluble factors and their roles in regenerative medicine. Curr Stem Cell Res Ther. 2010;5(2):103-10.

41. Sadat S, et al. The cardioprotective effect of mesenchymal stem cells is mediated by IGF-1 and VEGF. Biochem Biophys Res Commun. 2007; 363(3):674-9.

42. Rehman J, et al. Secretion of angiogenic and antiapoptotic factors by human adipose stromal cells. Circulation. 2004;109(10):1292-8.

43. Thomas D, et al. A shape-controlled tuneable microgel platform to modulate angiogenic paracrine responses in stem cells. Biomaterials. 2014 35(31):8757-66.

44. Mitchell KJ, et al. Identification and characterization of a non-satellite cell muscle resident progenitor during postnatal development. Nat Cell Biol. 2010;12(3):257-66

45. Joe AWB, et al. Muscle injury activates resident fibro/adipogenic progenitors that facilitate myogenesis. Nat Cell Biol. 2010;12(2):153-U144. 
46. Natarajan A, Lemos DR, Rossi FM. Fibro/adipogenic progenitors: a doubleedged sword in skeletal muscle regeneration. Cell Cycle. 2010;9(11):2045-6.

47. Yang JJ, et al. Human adipose tissue-derived stem cells protect impaired cardiomyocytes from hypoxia/reoxygenation injury through hypoxiainduced paracrine mechanism. Cell Biochem Funct. 2012;30(6):505-14.

48. Yang $D$, et al. The relative contribution of paracrine effect versus direct differentiation on adipose-derived stem cell transplantation mediated cardiac repair. PLoS One. 2013;8(3):e59020.

49. Hwang $\mathrm{JH}$, et al. Combination therapy of human adipose-derived stem cells and basic fibroblast growth factor hydrogel in muscle regeneration. Biomaterials. 2013;34(25):6037-45.

50. Bacou F, et al. Transplantation of adipose tissue-derived stromal cells increases mass and functional capacity of damaged skeletal muscle. Cell Transplant. 2004;13(2):103-11.

51. Pecanha $R$, et al. Adipose-derived stem-cell treatment of skeletal muscle injury. J Bone Joint Surg Am. 2012;94(7):609-17.

52. Pinheiro $\mathrm{CH}$, et al. Local injections of adipose-derived mesenchymal stem cells modulate inflammation and increase angiogenesis ameliorating the dystrophic phenotype in dystrophin-deficient skeletal muscle. Stem Cell Rev. 2012;8(2):363-74.

53. $\mathrm{Mu} \mathrm{X}$, et al. Mediators leading to fibrosis-how to measure and control them in tissue engineering. Oper Tech Orthop. 2010;20(2):110-8.

54. Lemos DR, et al. Nilotinib reduces muscle fibrosis in chronic muscle injury by promoting TNF-mediated apoptosis of fibro/adipogenic progenitors. Nat Med. 2015;21(7):786-94.

55. Burks TN, et al. Losartan restores skeletal muscle remodeling and protects against disuse atrophy in sarcopenia. Sci Transl Med. 2011;3(82):82ra37.

56. Shadrin IY, Khodabukus A, Bursac N. Striated muscle function, regeneration, and repair. Cell Mol Life Sci. 2016;73(22):4175-202.

57. Jarvinen TA, et al. Muscle injuries: biology and treatment. Am J Sports Med. 2005;33(5):745-64

58. Planat-Benard V, et al. Spontaneous cardiomyocyte differentiation from adipose tissue stroma cells. Circ Res. 2004;94(2):223-9.

59. Oh EJ, et al. In vivo migration of mesenchymal stem cells to burn injury sites and their therapeutic effects in a living mouse model. J Control Release. 2018;279:79-88.

60. Ahn SY, et al. Vascular endothelial growth factor mediates the therapeutic efficacy of mesenchymal stem cell-derived extracellular vesicles against neonatal hyperoxic lung injury. Exp Mol Med. 2018:50(4):26.

61. Zhang $L$, et al. Therapeutic effect of human umbilical cord-derived mesenchymal stem cells on injured rat endometrium during its chronic phase. Stem Cell Res Ther. 2018;9(1):36.

\section{Ready to submit your research? Choose BMC and benefit from:}

- fast, convenient online submission

- thorough peer review by experienced researchers in your field

- rapid publication on acceptance

- support for research data, including large and complex data types

- gold Open Access which fosters wider collaboration and increased citations

- maximum visibility for your research: over $100 \mathrm{M}$ website views per year

At BMC, research is always in progress.

Learn more biomedcentral.com/submissions 